\title{
An Accurate Facial Component Detection Using Gabor Filter
}

\author{
K. Sudhakar, P. Nithyanandam* \\ School of Computer Science and Engineering, VIT University, Chennai, TamilNadu, India \\ ${ }^{*}$ Coressponding author, e-mail: deancc.scse@vit.ac.in
}

\begin{abstract}
Face detection is a critical task to be resolved in a variety of applications. Since faces include various expressions it becomes a difficult task to detect the exact output. Face detection not only play a main role in personal identification but also in various fields which includes but not limited to image processing, pattern recognition, graphics and other application areas. The proposed system performs the face detection and facial components using Gabor filter. The results show accurate detection of facial components
\end{abstract}

Keywords: face detection, Gabor jets, Gabor Wavelet, facial components

\section{Introduction}

There is a need to detect faces to avoid fraud happening in day to day life and particularly in bank frauds. Detecting face should be accurate and excellent techniques has to be implemented to avoid such kind of frauds.

There are many techniques available to detect faces including Linear discriminant analysis, Principal component analysis, Local Binary Pattern etc. But these methods are having some limitation because the face image contains different lighting conditions, different poses and different expression. Due to these limitations, an accurate Facial component detection has been failed and facial recognition accuracy has been reduced. Chances of frauds, terrorism, bank robbery related activity has been increased because of false face recognition methods. To overcome these problems an efficient face detector is required. The proposed system provides the efficient face detector including accurate facial component detection. It uses step by step detector to detect the faces and Gabor filter is used to detect facial components.

Gabor filter is widely used in many area including image processing, pattern recognition, texture analysis, edge detection, feature extraction and face detection. Gabor filter provides an accurate facial component detection by avoiding different illumination, different poses and different expressions.

Abhishree et al. [1] has discussed on feature extraction done by using Gabor filter that can be combined with Anisotropic diffusion based pre-processing techniques and it can be used for face recognition system. But the computational speed is increased in this method. Xin Fan et al. [2] has investigated on fiducial point that can be detected by using standard gradient descent techniques .Various changes in faces are also identified in this method. In that edges of facial components are detected. But this method provides less accuracy when testing of different poses and expressions.

\section{Related Work}

Junjie Yanet et al. [3] has discussed face detection by structural models. In that the major Problem in face detection with local appearance and global appearance are handled. Local appearance deals with facial expressions and global appearance deals with facial poses. Advantage of structural model is to handle face with different expression and different poses. But accuracy is very less in the structural model.

Neslihan Koseet et al. [4] has investigated about face recognition done with mask spoofing. 2D and 3D mask database are tested using Counter measures. The analysis is done with different texture, shape and reflectance for the data set. Advantage of proposed system is 
the protection of face recognition systems against mask attack. Disadvantage of the above system is, it can be tested with polychrome mineral powder mask and we need to test with different materials.

Jacek Naruniec [5] has discussed about the face detection and facial feature detection done by using Discrete area filters. In that four facial components has been detected and it focus on fiducial point detection of facial components. But the detection is slow because it performs lot of analysis with facial components.

Akshay Bhatiaet et al. [6] proposed the face detection techniques using fuzzy concepts combined with skin colour. The test image is passed in the fuzzy classifier and faces are detected and HSV model are used to detect the skin colour.

M. Ramze Rezaeeet et al. [7] deals with fuzzy feature selection based on fuzzy technique fuzzy classification are used. Various feature description on image processing are described in this approach that includes Region-Centroid in the column, row and pixel, density, mean and standard deviation.

Detection of moving objects using fuzzy is proposed in Pojala Chiranjeeviet et al. [8] objects are detected in dynamically changing background. Background subtraction algorithms are used for the classification. Automatic face detection based on colour has been discussed. Types of face detection are Knowledge, Feature, Template and Appearance.

Mengjuan Chenlet et al. [9] discuses on image segmentation based on local region. It deals with quality metrics such as PSNR, MSF are calculated for analysing the performance. Segmentation of endometrial cancer has been proposed in Lidiya Lilly Thampi et al. [10]. In that the Segmentation is applied and the image is converted into multiple segments. Then the Morphological operations such as erosion and deletion are applied to the image for object detection. Junlin $\mathrm{Hu}$ et al. [11] has investigated the problem on fine grained face verification and its difficulties. The proposed system uses this dataset for testing. Rubel Biswas et al. [12] have proposed a system for detection and recognition of iris using Hough transformation for object detection and SVM classifiers are used for training. Fatemeh Hourali et al. [13] have been discussed about ear recognition using Invariant transformed DCT.

The various facial detection techniques were discussed including structural model, spatio-temporal analysis model, mask spoofing model, geometric based face recognition and other models provides the less accuracy. The proposed system for face detection using Gabor filter provides more accuracy.

\section{Proposed Approach}

The proposed system architecture are depicted in Figure 1. The proposed system takes input as image and it is fed into the face detector. The face detector detects whether the input image contains face or not. The face detection uses step by step detection techniques because it performs very fast and accurate detection for real-time object.

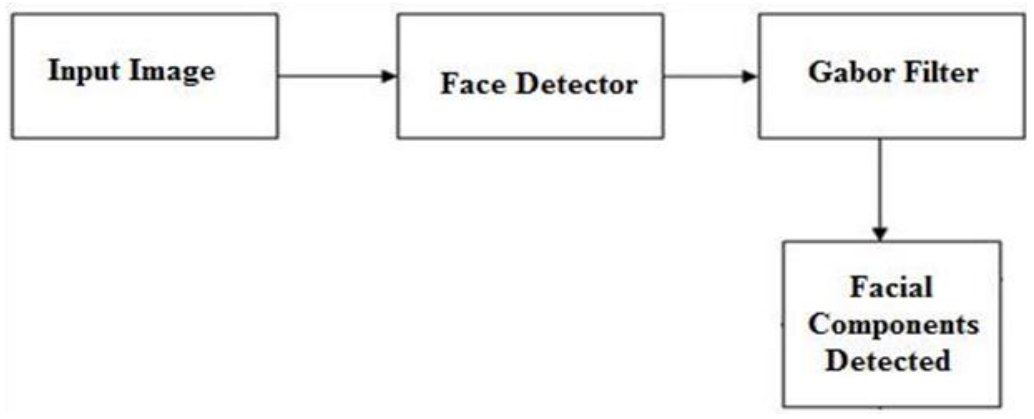

Figure 1. Face component detection system architecture

In face detection steps, first it performs the normalization which resize and perform rotation on faces. Then, it identifies facial similar features. All human faces share some similar properties and it is used to construct certain features. 
The properties that are similar for a human face are given below:

- The eyes region is like ellipse shape and inside round black region.

- The nose region is in triangle shape.

- Mouth is in three lines with skin color.

Based on common features the face has been detected and it passes into Gabor filter. Gabor filter generates the real part and imaginary part of given input image and then the facial components are detected. The proposed system of the Gabor filter in the spatial domain [2627], that has been expressed in the equation 1.

$$
\mathrm{G}\left(\mathrm{a}, \mathrm{b}, \theta_{1}, \mathrm{v}, \sigma_{1}\right)=\frac{1}{2 \Pi \sigma_{1}{ }^{2}} \exp \left|-\frac{a^{2}+b^{2}}{2 \sigma_{1}^{2}}\right| \exp \left\{2 \pi\left(v a \cos \theta_{1}+v b \sin \theta_{1}\right)\right\}
$$

where; $\mathrm{i}=\sqrt{ }-1$

$v$ is the frequency of the sinusoidal wave

$\theta 1$ controls the orientation of the function

61 is the standard deviation of the Gaussian envelope

Feature point of feature vectors are generated by using Gabor wavelet transform. The $\mathrm{k} 1$ th feature vector of $\mathrm{mth}$ reference face is defined in equation 2 .

$$
\mathrm{Vm}, \mathrm{k} 1=\{\mathrm{ak} 1, \mathrm{bk} 1, \mathrm{Rm}, \mathrm{n}(\mathrm{ak} 1, \mathrm{bk} 1) \mathrm{n}=1, \ldots ., 40\}
$$

where there are 40 Gabor filter,

Feature vector have 42 components,

The first two components represents in $(a, b)$ coordinates.

\section{Result and Discussion}

The input image used for the proposed system is depicted in Figure 2 Once the face is detected by using step by step face detector, the image results are passed into Gabor filter.

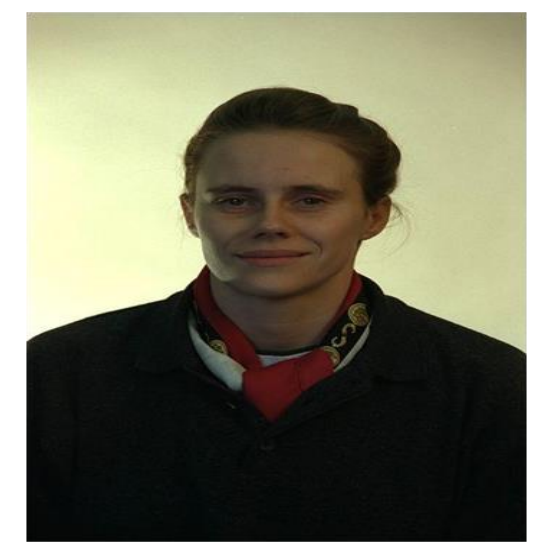

Figure 2. Input image

Gabor filter generates 40 wavelets. Each wavelet contains real and imaginary components. It uses five frequencies and eight orientations. Figure 3 describes the different magnitude and Figure 4 describes the real part generated by Gabor filter. 


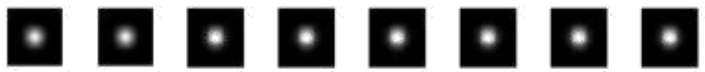

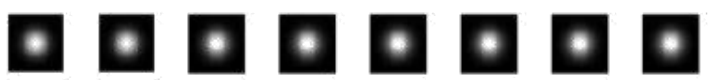

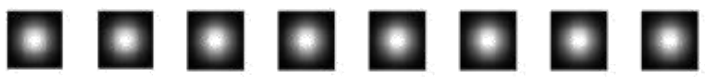 \\ ㅁㅁㅁㅁ

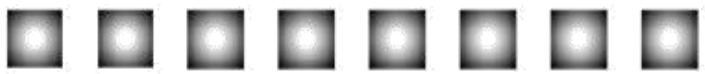

Figure 3. Magnitude part of input image

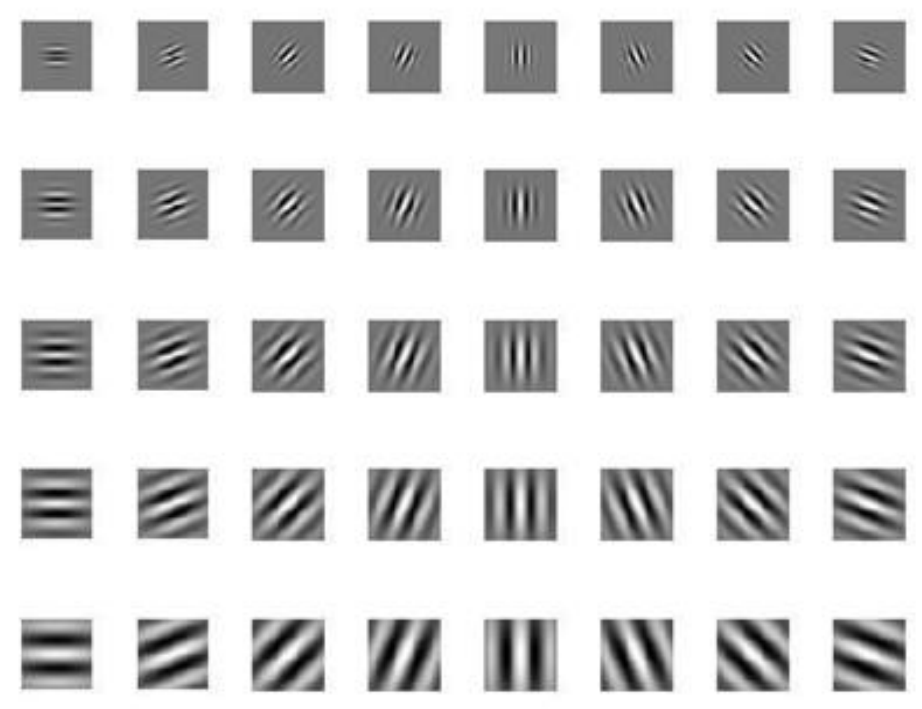

Figure 4. Real part of input image

Gabor filter is used to extract the features from input image. It creates Gabor array that contain Gabor features of input image that generates the Gabor Filter bank. Gabor Filter bank contains 5 scales and 8 orientations which is used to generate the magnitude and real parts of input image. It describes across multiple frequencies and orientations. Figure 5 describes the different magnitude part using Gabor wavelet and Figure 6 describes the imaginary part generated by Gabor wavelet. 


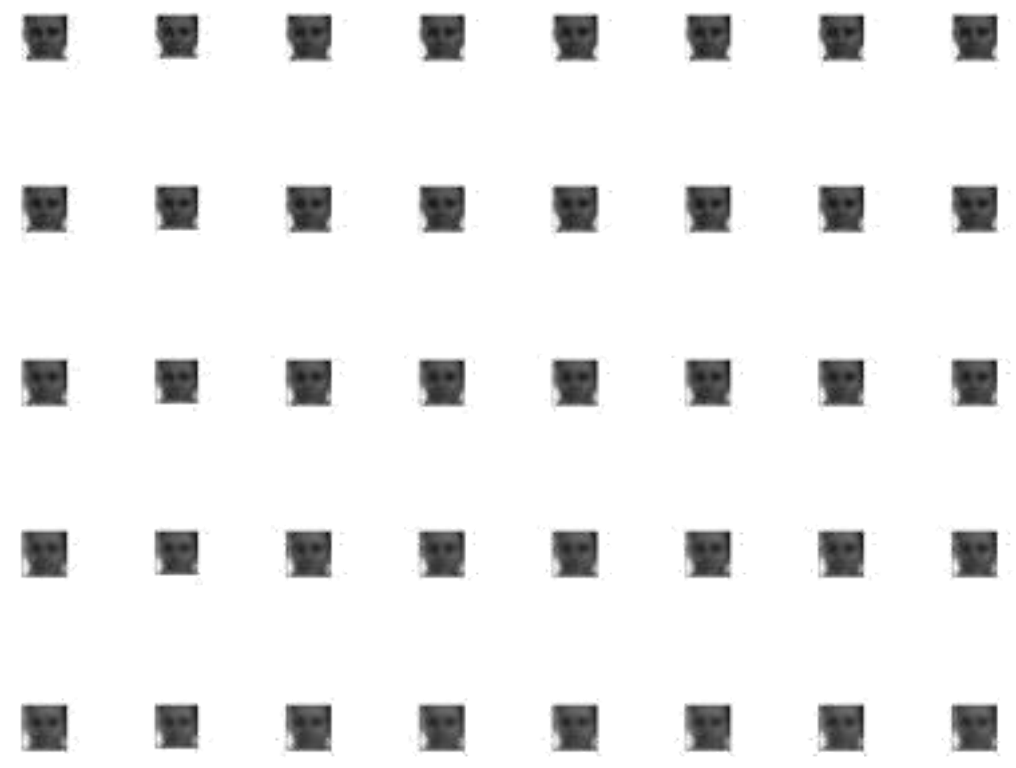

Figure 5. Magnitude part using Gabor wavelet

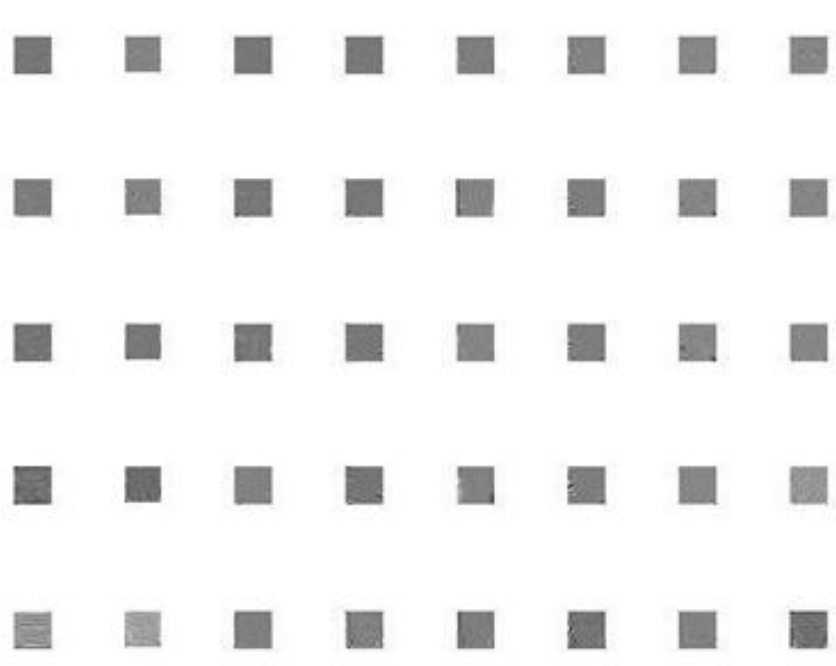

Figure 6. Imaginary part using Gabor wavelet

Figure 7 describes the face and facial components has been detected. The facial components includes left eye, right eye, nose, mouth etc. By using Gabor jets, the Fiducial point also been detected.

The proposed system has been tested with different input face images from Facial Recognition Technology database (FERET) [25-27] that contain different expression, different poses and Occluded faces. The sample tested images shown in Figure 8. 


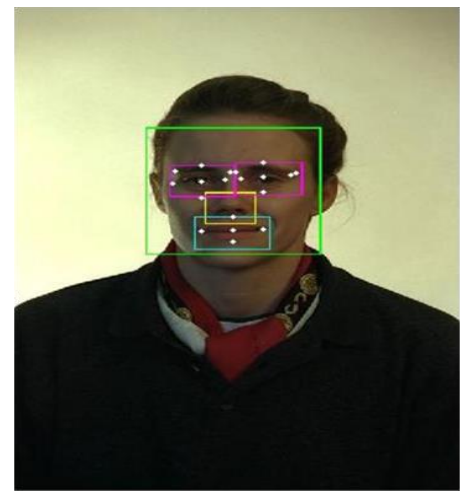

Figure 7. Face and facial components detected
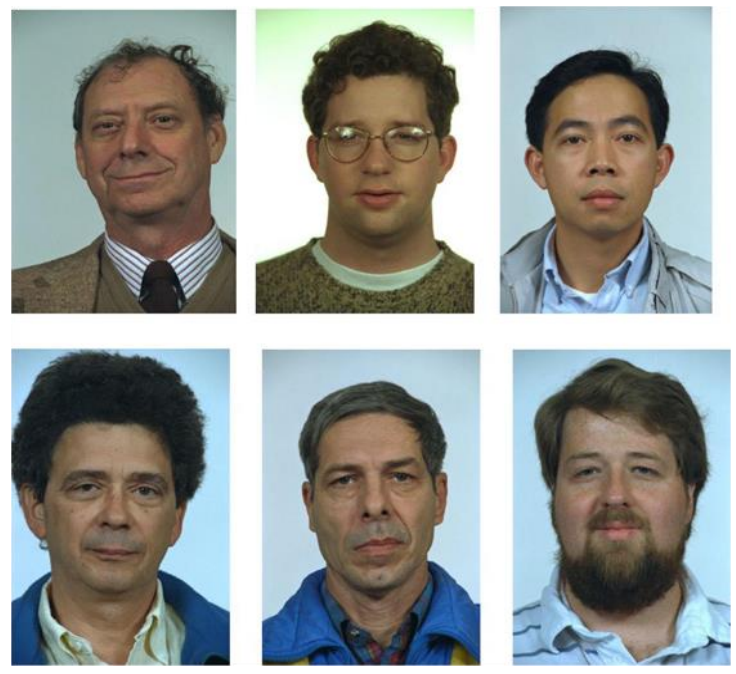

Figure 8. Different input images from FERET dataset with different expressions and occluded faces

Figure 9 describes that facial components are detected for the given sample images. In that facial components are identified and fiducial points also identified.
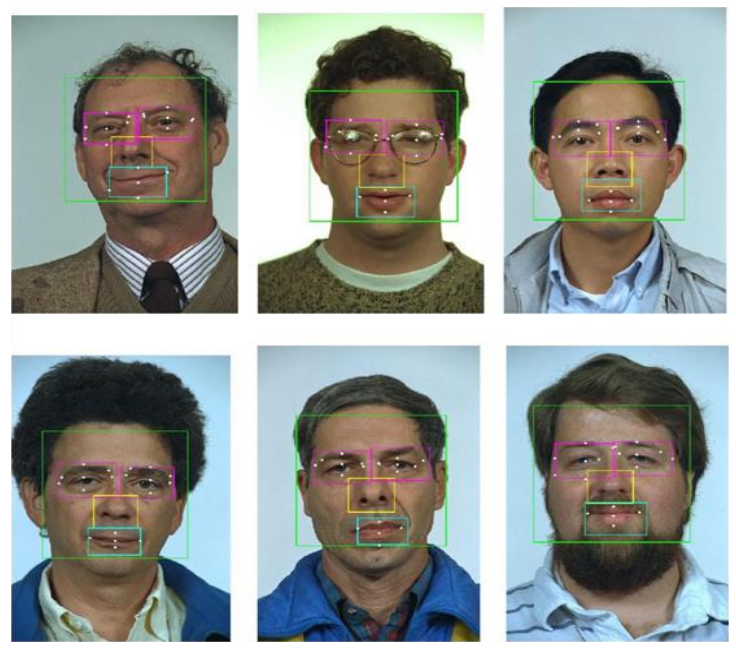

Figure 9. Fiducial Point detected in Different input images 
The comparison result between the proposed Gabor method with LBP and PCA method is defined in the Figure 10. The proposed method provides better recognition rate to detect face and facial components when compared with other methods. The proposed system provides efficient face recognition rate along with facial components detection which serves as a major advantage. The proposed method provides high security for the video surveillance area including banking, military and others.

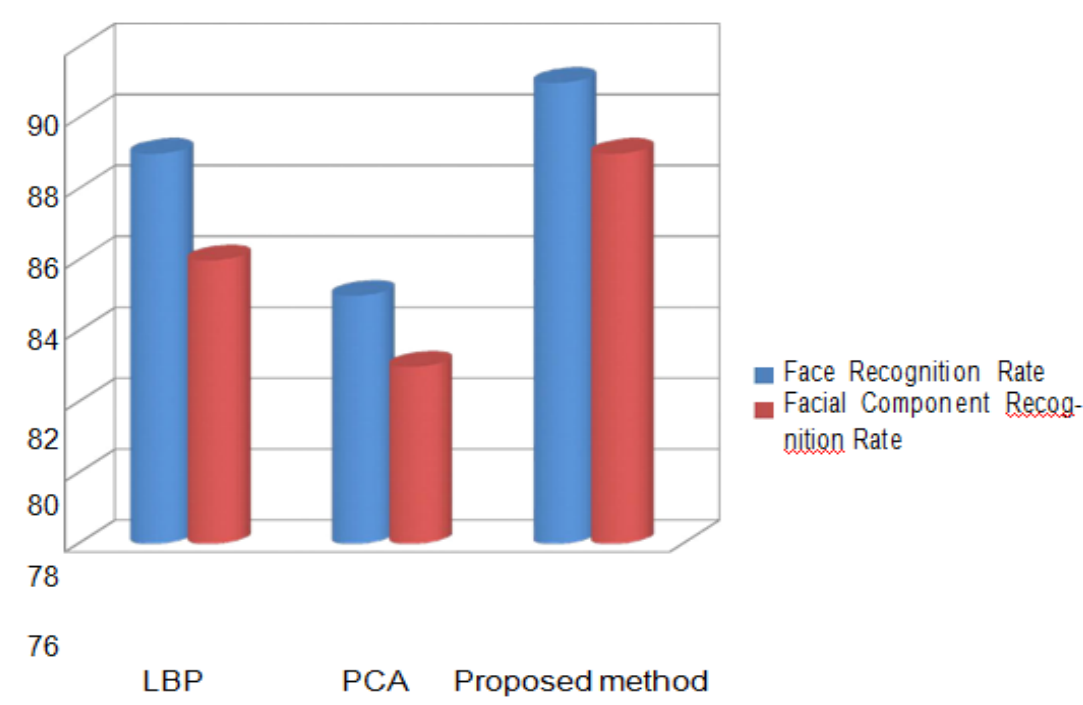

Figure 10. Recognition rate comparison chart between proposed and existing method

\section{Conclusion}

The proposed system has detected faces using Gabor filter. The facial components that includes left eye, right eye, nose, mouth has been detected and Fiducial points has been detected for each facial components using real and imaginary parts of Gabor filter. It can be tested with idle faces, face with different poses and different expressions. The occluded faces that contain different eye glasses are tested in the proposed method. The accuracy of facial components and fiducial point detection is high in our proposed method and can be used for various real time scenario applications.

The proposed system was tested with static images with glass occluded faces. In future it should be tested with dynamic images and also it should be implemented with other occluded face that includes facial mask and beard.

\section{References}

[1] Abhishree TM, Latha J, Manikandan K, Ramachandran S. Face Recognition using Gabor Filter based Feature Extraction with Anisotropic Diffusion as a pre-procsessing Technique. ElsevierProcedia Computer Science. ICATA. 2015

[2] Xin Fan, Hao Wang, ZhongxuanLuo, Yuntao Li, Wenyu Hu, DaiyunLuo. Fiducial Facial Point Extraction Using a Novel Projective Invariant. IEEE Transactions on Image Processing. 2015; 24(3).

[3] Junjie Yan, Xuzong Zhang, Zhen Lei, Stan Z Li. Face Detection by Structural Models. Image and Vision Computing. 2014; 32: 790-799.

[4] NeslihanKose, Jean-Luc Dugelay. Mask Spoofing in face recognition and countermeasures. ElsevierImage and Vision Computing. 2014; 32; 779-789.

[5] Jacek Naruniec. Discrete Area Filters in Accurate Detection of Faces and Facial Features. ElsevierImage and Vision Computing. 2014; 32: 979-993.

[6] Akshay Bhatia, SmritiSrivastava, Ankit Agarwal. Face Detection using Fuzzy Logic and Skin Color Segmentation in Images. Proc. Third International Conference on Emerging Trends in Engineering and Technology. 2010 
[7] RamzeRezaee, M Goedhart, B Lelieveldt BPF, Reiber JHC. Fuzzy Feature Selection. Pattern Recognition Society. Published by Elsevier Science Ltd. 1999

[8] Pojala Chiranjeevi, Somnath Sengupta. Neighborhood Supported Model Level Fuzzy Aggregation for Moving Object Segmentation. IEEE Transactions on Image Processing. 2014; 23(2).

[9] Mengjuan Chenl, Jianwei Li2, Hanqing Zhaol, Xiao Mal. A Local Region-based Level Set Algorithm for Image Segmentation. IEEE Workshop on Electronics, Computer and Applications. 2014

[10] Lidiya Lilly Thampi, Malarkhodi S. An Automatic Segmentation of Endometrial Canceron Ultrasound Images. Proc. International Conference on Communication and Signal Processing. 2013.

[11] Junlin Hu, Jiwen Lu, Yap-Peng Tan. Fine-Grained Face Verification: Dataset and Baseline Results. Proc. of International conference on Biometrics (ICB) 2015.

[12] Rubel Biswas, Jia Uddin, Md Junayed Hasan. A New Approach of Iris Detection and Recognition. International Journal of Electrical and Computer Engineering (IJECE). 2017; 7(5).

[13] Fatemeh Hourali, Sorayya Gharravi. An Ear Recognition Method Based on Rotation Invariant Transformed DCT. International Journal of Electrical and Computer Engineering (IJECE). 2017; 7(5).

[14] Paul Viola, Michael Jones. Robust Real Time Face Detection. Proc. Second International Workshop on Statistical and Computational Theories of Vision-modeling, Learning, Computing and Sampling. 2001: 1-25.

[15] Hjelmas E, Low BK. Face Detection: A Survey. Computer Vision and Image Understanding. 2001; 83(3): 236-274.

[16] Visal Kith, Mohamed El-Sharkawy, Tonya Bergeson-Dana, Salwa El-Ramly, Said El Noubi. A Feature and Appearance Method for Eye Detection on Gray Intensity Face Images. Computer Engineering \& Systems, ICCES. 2008: 21-25.

[17] Suzuki Y, Shibata T. Multiple-Clue Face Detection Algorithm Using Edge Based Feature Vectors. IEEE transaction on Acoustics, Speech, and Signal, Processing. 2004; 5: 35-35.

[18] Rowley HA, Baluja S, Kanade T. Neural Network-based Face Detection. IEEE transaction on Pattern Analysis and Machinelntelligence. 1998; 20: 23-38.

[19] Devijver PA, Kittler J. Pattern Recognition: A Statistical Approach, Prentice-Hall, Englewood Cli!s, NJ. 1982

[20] Quinlan JR., Introduction of decision trees, in: Machine Learning, Kluwer Acadamic Publishers, Dordrecht. 1986: 81-106.

[21] Baim PW. A Method for Attribute Selection in Inductive Learning Systems. IEEE Trans. Pattern Anal. Mach. Intell. 1988; 10(6): 888-896.

[22] Rauber TW, Steiger-Garcao AS. Feature Selection of Categorical Attributes Based on Contingency Table Analysis. Proc. of the 5th Portuguese Conference on Pattern Recognition, Porto, Portugal. 1993.

[23] Pal SK, Chakraborty B. Fuzzy Set Theoretic Measure for Automatic Feature Evaluation. IEEE Trans. Systems. ManCybernet. 1986; 16(5): 754-760.

[24] Cristani, M, Farenzena M, Bloisi D, Murino, V., "Background Subtraction for Automated Multi-sensor Surveillance: A comprehensive review. EURASIP J. Adv. Signal Process. 2010: 20-25.

[25] Color FERET: http://www.nist.gov/itl/iad/ig/colorferet/cfm

[26] PJ Phillips, H Wechsler, J Huang, P Rauss. The FERET Database and Evaluation Procedure for Face Recognition Algorithms. Image and Vision Computing J. 1998; 16(5): 295-306.

[27] PJ Phillips, H Moon, SA Rizvi, PJ Rauss. The FERET Evaluation Methodology for Face Recognition Algorithms. IEEE Trans. Pattern Analysis and Machine Intelligence. 2000; 22: 1090-1104.

[28] AP Ginsburg. Visual Information Processing Based on Spatial Filters Constrained by Biological Data. AMRL tech. Rep. 1978: 78-129.

[29] AG Goldstein. Facial Feature Variation: Anthropometric Data II. Bull. Psychonomic Soc. 1979; 13: 191-193.

[30] Burcu Kepenekci, Gozde Bozdagi Akar. Motion Analysis Using 3D Gabor Kernels. IEEE 16th Signal Processing, Communication and Applications Conference. April 2008.

[31] Jacek Naruniec. Discrete Area Filters in Accurate Detection of Faces and Facial Features. Elsevier: Image and Vision Computing. 2014; 32: 979-993. 